Pacific Journal of Mathematics

COMPACTOID AND COMPACT FILTERS

C Gabriel 


\section{COMPACTOID AND COMPACT FILTERS}

\section{Szymon Dolecki, Gabriele H. Greco and Alojzy LeChicki}

We study compactoid and compact filters which generalize the concepts of convergent filters and compact sets. In particular, we investigate their properties in subregular and regular spaces, their localizations, and their countable variants. Several classical results follow (e.g., theorems of Tychonoff, Kuratowski, Choquet). More recent results on preservation of compactness (e.g., Smithson) and local compactness (e.g. Lambrinos) are extended and refined.

0. Introduction. Several topological properties of relations may be expressed in terms of some filters on the image space. Therefore, certain investigations of relations may be reduced to the study of filters, thus simplifying the arguments. There arise compactoid, compact, semiconvergent, locally compact, cocompact, subregular, regular and other filters. Compactoid and compact filters generalize both convergent filters and compact sets. Frequently they may be used where convergence or compactness of sets is too strong an assumption (see, for instance, the classical Theorem 7.10 of Kuratowski [15]). Compactoid filters find numerous applications in optimization, generalized differentiation, differential equations, fixed point theory (see e.g. [20] and [2] where compactoid filters are hidden under the guise of measure of noncompactness) and elsewhere, primarily in the context of existence results.

To our knowledge, the concept of compactoid filter appeared for the first time in [24] by Topsøe, formulated with the aid of nets in topological spaces. ${ }^{1}$ Unfamiliar with this, two of us reintroduced compactoid filters in [9]. The same idea occurred independently to Penot; in [20] he presents an extensive collection of applications. Going back to the twenties one finds compact sequences of Urysohn [25]. They constitute a sequential counterpart of compactoid filters.

Compactoid and compact filters are also of considerable theoretical interest. They provide a broader comprehension of the notion of compactness and enable one to establish new and subtler results (for instance, on preservation of compactness).

\footnotetext{
${ }^{1}$ The notion of compactoid filter appears also in M. P. Kac, Characterization of some classes of pseudotopological linear spaces, in Convergence Structures and Applications to Analysis, Akademie-Verlag, Berlin, 1980, 115-135.
} 
We found it natural to carry out our study of compactoid filters in pseudotopological spaces. This enables us to better see their nature and to distinguish topological properties from pretopological and pseudotopological ones. Later we discovered an intimate and essential relationship between pseudotopologies and compactness [8].

A filter is compactoid if every ultrafilter of it converges. Compactoid and compact sets are special cases of compactoid and compact filters. We establish the relationship between compactoid and overcoverable filters. It is demonstrated that if $\mathscr{F}$ is compactoid (compact) in a pretopology $\pi$, then its neighborhood filter $\mathscr{N}(\mathscr{F})$ is comopactoid (compact) in $\pi^{2}$.

A filter is subregular if its closure filter is compactoid. The adherence of every subregular filter is compactoid. In subregular spaces every compactoid filter is subregular. In regular spaces the closure of every convergent filter converges. This paper discusses regular spaces primarily in the context of compactness and compactoidness.

An extension of the Tychonoff theorem (given already in [20] in topological spaces) says that a filter in a product pseudotopological space is compactoid if and only if every projection of it is compactoid.

We refine and extend results of Smithson [23] on subcontinuous relations, and relations mapping compact sets into compact sets. It is shown that a relation is subcontinuous if and only if it maps every compactoid filter into a compactoid filter.

A filter $\mathscr{F}$ is pseudocompactoid if the lower limit along $\mathscr{F}$ of every lower semicontinuous function is greater than $-\infty$. We show that every countably compactoid filter is pseudocompactoid.

In Dieudonné complete topological spaces, $\mathscr{F}$ is finer than the neighborhood filter of a closed set $A$ if and only if the trace of $\mathscr{F}$ on the complement of $A$ is compactoid and the $\mathscr{F}$-boundary of $A$ is a subset of $A$. An alternative result in metrizable spaces, but without the assumption that $A$ be closed, generalizes Choquet's [5, Thm 3].

1. Filters and Grills. This section has a preliminary character: its aim is to recall a few facts that we shall use later on. The basic reference is [4], from which we slightly differ in terminology and presentation.

For a nonvoid family of subsets of $X, \mathscr{A} \subset 2^{X}$, we define its conjugate $\mathscr{A}^{*}$ by

$$
B \in \mathscr{A}^{*}, \quad \text { iff } \quad B^{c} \notin \mathscr{A} .
$$

$\mathscr{A}^{* *}=\mathscr{A}$ and for a collection of families,

$$
\left(\bigcup_{i \in I} \mathscr{A}_{i}\right) *=\bigcap_{i \in I} \mathscr{A}_{i}^{*}, \quad\left(\bigcap_{i \in I} \mathscr{A}_{i}\right) *=\bigcup_{i \in I} \mathscr{A}_{i}^{*}
$$


The grill, $\mathscr{A} \#$, of $\mathscr{A}$ is the family of all subsets of $X$ intersecting every element of $\mathscr{A}$.

A family $\mathscr{A}$ is called based if $\varnothing \notin \mathscr{A}$ and $B \in \mathscr{A}, B \subset A$ implies that $A \in \mathscr{A}$. A subfamily $\mathscr{B}$ of a based family $\mathscr{A}$ is a base of $\mathscr{A}(\mathscr{A}$ is generated by $\mathscr{B}$ ) if for every $A \in \mathscr{A}$ there is $B \in \mathscr{B}$ such that $B \subset A$. If $\mathscr{A}$ is based, then $\mathscr{A}^{\#}=\mathscr{A}^{*}$. A based family is called countably based if it has a countable base. We write $\mathscr{C} \leq \mathscr{A}(\mathscr{A}$ is finer then $\mathscr{C})$ if for every $C \in \mathscr{C}$ there is $A \in \mathscr{A}$ with $A \subset C$. The grill of a family is always based. $\mathscr{A}^{\#} \subset \mathscr{C}^{\#}$ if and only if $\mathscr{A}$ is finer than $\mathscr{C}$.

A nonvoid family $\mathscr{F} \subset 2^{X}$ is a filter whenever $\varnothing \notin \mathscr{F}$ and

$$
A \cap B \in \mathscr{F} \text { iff } A \in \mathscr{F} \text { and } B \in \mathscr{F} \text {. }
$$

A family $\mathscr{G}$ is a filter grill (i.e. the grill of some filter) if and only if

$$
A \cup B \in \mathscr{B} \text { iff } A \in \mathscr{G} \text { or } B \in \mathscr{G} \text {. }
$$

Denote by $\beta X$ the collection of all ultrafilters on $X$, by $\beta \mathscr{F}$ that of all ultrafilters finer than $\mathscr{F}$. A filter $\mathscr{U}$ is an ultrafilter if and only if $\mathscr{U}^{\#}=\mathscr{U}$.

Two filters $\mathscr{F}$ and $\mathscr{G}_{\text {meet }}$ if for every $F \in \mathscr{F}$ and $G \in \mathscr{G}, F \cap G \neq \varnothing$. Otherwise, they are disjoint. The supremum $\mathscr{F} \vee \mathscr{G}$ exists if and only if $\mathscr{F}$ and $\mathscr{G}$ meet; $\mathscr{F} \vee \mathscr{G}=\{F \cap G: F \in \mathscr{F}, G \in \mathscr{G}\}$. The discrete filter $\mathscr{N}_{\iota}(A)$ of a nonempty set $A$ is composed of all sets that include $A$. We say that $\mathscr{F}$ meets $A$ if $\mathscr{F}$ and $\mathscr{N}_{\iota}(A)$ meet. Then their supremum is denoted by $\mathscr{F} \vee A$ and called the trace of $\mathscr{F}$ on $A$.

Let $\Gamma$ be a relation in $X \times Y$. We also denote it $\Gamma: X \rightrightarrows Y$. As usual for $A \subset X, \Gamma A=\{y:(x, y) \in \Gamma, x \in A\}$. For a filter $\mathscr{F}$ in $X$ the family $\Gamma \mathscr{F}=\{\Gamma F: F \in \mathscr{F}\}$ is a filter base if and only if $\Gamma^{-1} Y \in \mathscr{F} \#$, where $\Gamma^{-1}$ denotes the inverse relation. The resulting image filter of $\Gamma$ will also be denoted $\Gamma \mathscr{F}$. This is a slight abuse but will not cause confusion.

Mappings constitute special relations. Let $f: X \rightarrow Y$ be a mapping and let $\mathscr{A} \subset 2^{X}$. Then

$$
f(\mathscr{A})^{\#} \leq f\left(\mathscr{A}^{\#)}\right. \text {. }
$$

From this it is easily deduced that the image filter by a mapping of an ultrafilter is an ultrafilter.

2. Pseudotopologies, pretopologies, topologies. We recall here some basic facts from convergence theory. Most of them may be found in the fundamental work [5], a few others are extracted from [7]. Convergence theory is instrumental for the rest of this paper so we list useful facts, but 
do not try to display the underlying ideas. Several authors adopt a different terminology than [5] (see the monograph of Gähler [12]).

A pseudotopology $\theta$ in a set $X$ is a structure of $X$ which may be defined through a convergence relation $\operatorname{Lim}=\operatorname{Lim}^{\theta}$ that maps filters $\phi X$ (defined in $X$ ) to subsets of $X$ and which satisfies:

$$
x \in \operatorname{Lim} \mathscr{N}_{\iota}(x)
$$

(where $\mathscr{N}_{\iota}(x)=\{B \subset X: x \in B\}$ is the discrete filter of $x$ );

$$
\text { if } \mathscr{F} \subset \mathscr{G} \text {, then } \operatorname{Lim} \mathscr{F} \subset \operatorname{Lim} \mathscr{G} \text {; }
$$

if $x \notin \operatorname{Lim} \mathscr{F}$, then there is $\mathscr{G} \supset \mathscr{F}$ such that for every $\mathscr{H} \supset \mathscr{G}, x \notin \operatorname{Lim} \mathscr{H}$.

Given pseudotopologies $\sigma$ and $\theta$ we say that $\sigma$ is finer than $\theta(\theta$ is coarser than $\sigma$ ) if $\operatorname{Lim}^{\sigma} \subset \operatorname{Lim}^{\theta}$. We have, as a result of (2.2) (2.3),

$$
\operatorname{Lim} \mathscr{F}=\bigcap_{\mathscr{U} \in \beta \mathscr{F}} \operatorname{Lim} \mathscr{U} \text {. }
$$

The adherence of a filter $\mathscr{F}$ is defined by

$$
\text { Adh } \mathscr{F}=\bigcup_{\mathscr{U} \in \beta \mathscr{F}} \operatorname{Lim} \mathscr{U} \text {, }
$$

and one observes that for ultrafilters convergence and adherence coincide: if $\mathscr{U} \in \beta X$ then $\operatorname{Lim} \mathscr{U}=\operatorname{Adh} \mathscr{U}$. Define the closure of a set $A$ of $X$ as

$$
\mathrm{Cl} A=\operatorname{Adh} \mathscr{N}_{\iota}(A),
$$

where $\mathscr{N}_{\iota}(A)$ is the discrete filter of $A$, and define the pretopological adherence of a filter $\mathscr{A}$ on $X$ by

$$
\mathrm{Cl} \mathscr{A}=\bigcap_{A \in \mathscr{A}} \mathrm{Cl} A .
$$

We always have that, for a filter $\mathscr{F}$,

$$
\text { Adh } \mathscr{F} \subset \mathrm{Cl} \mathscr{F} \text {. }
$$

The closure filter $\overline{\mathscr{F}}$ of a filter $\mathscr{F}$ is the filter generated by $\{\mathrm{Cl} F$ : $F \in \mathscr{F}\}$.

The operation $\mathrm{Cl}: 2^{X} \rightarrow 2^{X}$ satisfies the properties $\mathrm{Cl} \varnothing=\varnothing, A \subset$ $\mathrm{Cl} A$ and $\mathrm{Cl}(A \cup B)=\mathrm{Cl} A \cup \mathrm{Cl} B$; hence for every $x \in X$ the collection $\{A: x \in \mathrm{Cl} A\}$ is a filter grill and its filter $\mathscr{N}(x)=\mathscr{N}_{\theta}(x)$, called the neighborhood filter of $x$ (in $\theta$ ), has the property that for every $N \in \mathscr{N}(x)$, $x \in N$. Note that the neighborhood filter of $x$ is equal to the intersection of all the filters convergent to $x$.

The interior of a set $A$ is equal to

$$
\operatorname{Int}_{\theta} A=\left(\mathrm{Cl}_{\theta} A^{c}\right)^{c},
$$

where, as usual, ${ }^{c}$ means complement. 
Observe the equivalences:

$$
\begin{aligned}
& Q \in \mathcal{N}(x), \\
& x \in \operatorname{Int} Q, \\
& x \notin \mathrm{Cl} Q^{c} .
\end{aligned}
$$

Given a subset $A$ of $X$ we say that $Q$ is a neighborhood of $A$ if $A \subset$ Int $Q$. The set $\mathscr{N}(A)=\{Q \subset X: A \subset$ Int $Q\}$ is the neighborhood filter of $A$ (provided $A$ is not empty), and for a family $\mathscr{A}$ of subsets of $X$ the neighborhood of $\mathscr{A}$ is

$$
\mathscr{N}(\mathscr{A})=\bigcup_{A \in \mathscr{A}} \mathscr{N}(A)
$$

One notes that

$$
Q \in \mathscr{N}(A), \quad \text { iff } \quad \mathrm{Cl} Q^{c} \cap A=\varnothing .
$$

LeMma 2.1. An $H$ belongs to $\mathscr{N}(\mathscr{F})^{\#}$ if and only if $\mathrm{Cl} H$ is in $\mathscr{F} \#$.

Proof. $H \in \mathscr{N}(\mathscr{F})^{\#}$ whenever $H^{c} \notin \mathscr{N}(\mathscr{F})$, that is, if and only if Int $H^{c} \notin \mathscr{F}$, that is, $\mathrm{Cl} H \in \mathscr{F}^{\sharp}$.

CoROllaRy 2.2. Let $\mathscr{F}$ and $\mathscr{H}$ be filters. $\mathscr{H}$ meets $\mathscr{N}(\mathscr{F})$ if and only if $\overline{\mathscr{H}}$ meets $\mathscr{F}$.

A subset $B$ of $X$ is called open whenever Int $B=B$. A set is closed provided it is the complement of an open set. The family of open subsets of a pseudotopological space satisfies the axioms of the open subsets of a topological space.

A pseudotopology $\pi$ is called a pretopology if for every $x$ the neighborhood filter of $x$ converges to $x$ :

$$
x \in \operatorname{Lim}^{\pi} N_{\pi}(x) .
$$

In other words, $\pi$ is a pretopology if and on ly if $x \in \operatorname{Lim}^{\pi} \mathscr{F}$ amounts to $\mathscr{F} \supset \mathscr{N}_{\pi}(x)$.

A pseudotopology $\pi$ is a pretopology if and only if for every filter $\mathscr{F}$,

$$
\operatorname{Adh}^{\pi} \mathscr{F}=\mathrm{Cl}_{\pi} \mathscr{F} .
$$

A pretopology is called Hausdorff if the neighborhood filters of each two distinct points are disjoint.

A pretopology $\tau$ is a topology if for every $x$ and each $Q \in \mathscr{N}_{\tau}(x)$ there is an open set $B$ such that $x \in B \subset Q$. Given a pretopology $\pi$ we define 
its square $\pi^{2}$ as the pretopology in which the neighborhood filters are

$$
\mathscr{N}_{\pi^{2}}(x)=\mathscr{N}_{\pi}\left(\mathscr{N}_{\pi}(x)\right) \text {. }
$$

In this pretopology $x \in \operatorname{Lim}^{\pi^{2}} \mathscr{F}$ if and only if $\mathscr{F}$ is finer than $\mathscr{N}_{\pi^{2}}(x)$. We observe that for every set $A$,

$$
\mathrm{Cl}_{\pi^{2}} A=\mathrm{Cl}_{\pi}\left(\mathrm{Cl}_{\pi} A\right) \text {. }
$$

A pretopology $\tau$ is a topology if and only if $\tau^{2}=\tau$.

Let $Z$ be a subset of a pseudotopological space $X$. The induced pseudotopology on $Z$ is defined by associating with every filter $\mathscr{F}$ on $Z$, $\operatorname{Lim}_{Z} \mathscr{F}=\operatorname{Lim} \mathscr{F} \cap Z$.

Let $X, Y$ be pseudotopological spaces and let $A: X \rightarrow Y$ be a mapping. We say that $A$ is continuous at $x$ if for every filter $\mathscr{F}$ in $X$ such that $x \in \operatorname{Lim} \mathscr{F}, A(x) \in \operatorname{Lim} A \mathscr{F}$.

3. Compactoid and overcoverable filters. Compact filters. Let $X$ be a pseudotopological space. A filter $\mathscr{F}$ in $X$ is said to be compactoid whenever for every ultrafilter $\mathscr{U}$ finer than $\mathscr{F}, \operatorname{Lim} \mathscr{U} \neq \varnothing$.

A nonvoid subset $A$ of $X$ is compactoid if its discrete filter $\mathscr{N}_{\iota}(A)=\{B$ : $A \subset B\}$ is compactoid. In other words, $A$ is compactoid if and only if for each ultrafilter $\mathscr{U}$ such that $A \in \mathscr{U}, \operatorname{Lim} \mathscr{U} \neq \varnothing$.

This definition of compactoid sets coincides with that given in [5]. The concept of compactoid set has been rediscovered by some authors in the framework of topological spaces (see, e.g., "bounded sets" of [16]). Compactoid filters were previously called "compact" ([9] [20]) but we reserve the latter term for another notion.

We say that $\mathscr{F}$ is $H$-compactoid, or compactoid with respect to a set $H$ provided that for each ultrafilter $\mathscr{U}$ of $\mathscr{F}, H \cap \operatorname{Lim} \mathscr{U} \neq \varnothing$.

The above notion is different from that of $\mathscr{F}$ being compactoid on $H$ (which means that the restriction of $\mathscr{F}$ to $H$ is compactoid in the pseudotopology induced on $H$. Indeed the latter means that for every $\mathscr{U}$ finer than $\mathscr{F} \vee H, H \cap \operatorname{Lim} \mathscr{U} \neq \varnothing)$.

A filter is compactoid if and only if it is compactoid with respect to the whole space. A filter compactoid with respect to $G$ is also compactoid with respect to every $H \supset G$. If a filter is $H$-compactoid for a pseudotopology $\sigma$ and $\theta$ is coarser than $\sigma$, then it is $H$-compactoid for $\theta$. We observe that the intersection of two compactoid (with respect to $H$ ) filters is compactoid (with respect to $H$ ). Of course, if $\mathscr{F}$ is $H$-compactoid and $\mathscr{G} \supset \mathscr{F}$, then $\mathscr{G}$ is $H$-compactoid. Therefore every locally compactoid filter (that is, such that it contains a compactoid set) is compactoid. 
PROPOSITION 3.1. We note the equivalence of:

(i) $\mathscr{F}$ is compactoid with respect to $H$.

(ii) For every $\mathscr{G}$ that meets $\mathscr{F}$, Adh $\mathscr{G} \cap H \neq \varnothing$.

(iii) For every $\mathscr{G}$ that meets $\mathscr{F}, \operatorname{Adh} \mathscr{G} \cap$ Adh $\mathscr{F} \cap H \neq \varnothing$.

Proof. (i) $\Rightarrow$ (iii). Let $\mathscr{G}$ meet $\mathscr{F}$. Then there is an ultrafilter $\mathscr{U}$ finer than $\mathscr{G} \vee \mathscr{F}$. Thus

$$
\operatorname{Lim} \mathscr{U}=\operatorname{Adh} \mathscr{U} \subset \operatorname{Adh}(\mathscr{G} \vee \mathscr{F}) \subset \operatorname{Adh} \mathscr{G} \cap \operatorname{Adh} \mathscr{F},
$$

and since $\mathscr{U} \supset \mathscr{F}, \operatorname{Lim} \mathscr{U} \cap H \neq \varnothing$; (iii) follows. Obviously (iii) $\Rightarrow$ (ii). To prove (ii) $\Rightarrow$ (i) note that each ultrafilter finer than $\mathscr{F}$ meets $\mathscr{F}$.

COROLLARY 3.2. A filter $\mathscr{F}$ is compactoid if and only if, for every filter $\mathscr{G}$ it meets,

$$
\text { Adh } \mathscr{F} \cap \text { Adh } \mathscr{G} \neq \varnothing .
$$

We conclude that $\mathscr{F}$ is compactoid if and only if it is compactoid with respect to Adh $\mathscr{F}$. Moreover, if a space has the property that the limit of every filter is at most a singleton, and if $\mathscr{F}$ is compactoid with respect to a set $H$, then Adh $\mathscr{F} \subset H$.

A filter is $\mathscr{H}$-compactoid (compactoid with respect to (a family of sets) $\mathscr{H})$ if it is $H$-compactoid for every $H \in \mathscr{H}$.

THEOREM 3.3. Let $\pi$ be a pretopology and let $\mathscr{F}, \mathscr{G}, \mathscr{H}$ be filters. If $\mathscr{F}$ is $\mathscr{G}$-compactoid and $\mathscr{G}$ is $\mathscr{H}$-compactoid (both in $\pi$ ), then $\mathscr{F}$ is $\mathscr{H}$-compactoid in $\pi^{2}$

Proof. Let $\mathscr{K}$ be a filter that meets $\mathscr{F}$. Then by the $\mathscr{G}$-compactoidness of $\mathscr{F}, \mathscr{G}$ meets Adh $\mathscr{K}$. In other words, the filter $\mathscr{G} \vee$ Adh $\mathscr{K}$ exists (and, of course, meets $\mathscr{G})$. Since $\mathscr{G}$ is $\mathscr{H}$-compactoid, $\operatorname{Adh}(\mathscr{G} \vee \operatorname{Adh} \mathscr{K})$ belongs to $\mathscr{H}^{\#}$ (that is, meets $\mathscr{H}$ ). We have

$$
\varnothing \neq \operatorname{Adh}^{\pi}\left(\mathscr{G} \vee \operatorname{Adh}^{\pi} \mathscr{K}\right) \subset \operatorname{Adh}^{\pi} \mathscr{G} \cap \mathrm{Cl}_{\pi}\left(\operatorname{Adh}^{\pi} \mathscr{K}\right),
$$

and since

$$
\mathrm{Cl}_{\pi}\left(\operatorname{Adh}^{\pi} \mathscr{K}\right) \subset \operatorname{Adh}^{\pi^{2}} \mathscr{K},
$$

we infer that $\operatorname{Adh}^{\pi^{2}} \mathscr{K}$ is in $\mathscr{H}^{\#}$ and thus $\mathscr{F}$ is $\mathscr{H}$-compactoid in $\pi^{2}$.

We infer that in topological spaces the property of being compactoid with respect to another filter is transitive. 
We are now in a position to define compact filters. A filter is said to be compact if it is compactoid with respect to itself. Accordingly, $\mathscr{F}$ is compact if and only if for every ultrafilter $\mathscr{U} \supset \mathscr{F}, \operatorname{Lim} \mathscr{U} \in \mathscr{F} \#$. Every compact filter is compactoid.

We observe that in pretopological spaces the neighborhood filter of every point is compact.

A nonempty subset $A$ of a pseudotopological space is called compact if its discrete filter is compact, that is, if every ultrafilter $\mathscr{U}$ with $A \in \mathscr{U}$ has a limit point in $A$. In other words, a subset of a pseudotopological space is compact if and only if it is compactoid in the induced pseudotopology. A space $X$ is compact if and only if the filter $\{X\}$ is compact (or compactoid). A subset of a compact space is compactoid but not necessarily compact. A compactoid closed subset of a pseudotopological space is compact.

Our definition of compact filters is thus compatible with the usual compactness of sets.

As a result of Proposition 3.1, we have

COROLlaRy 3.4. A filter $\mathscr{F}$ is compact if and only if for every filter $\mathscr{G}$ that meets $\mathscr{F}$, Adh $\mathscr{G} \in \mathscr{F} \sharp$ or, equivalently, Adh $\mathscr{G} \cap \operatorname{Adh} \mathscr{F} \in \mathscr{F} \sharp$.

Consequently every compactoid closed set is compact. We recall that in the spaces with unicity of limits a filter which is $H$-compactoid satisfies Adh $\mathscr{F} \subset \boldsymbol{H}$. Therefore, if $\mathscr{F}$ is a compact filter in such a space then Adh $\mathscr{F} \subset \bigcap_{F \in \mathscr{F}} F$. In a Hausdorff pretopological space every compact set is closed.

Notice, as well, that the coarser the pseudotopology the more compact filters it has. The intersection of two compact filters is compact.

A family is a cover of a set if the union of its elements includes this set. We say that $\mathscr{Q} \subset 2^{X}$ is an overcover of a set $A$ if

$$
A \subset \bigcup_{Q \in \mathscr{Q}} \text { Int } Q \text {. }
$$

We single out two lemmas which constitute the core of many arguments concerning compactness. Define $\mathscr{Q}^{c}=\left\{Q^{c}: Q \in \mathscr{Q}\right\}$.

LEMMA 3.5. A family 2 is not an overcover of $A$ if and only if

$$
A \cap \mathrm{Cl} \mathscr{Q}^{c} \neq \varnothing \text {. }
$$

LEMMA 3.6. No finite subfamily of $\mathscr{Q}$ is a cover of $A$ if and only if $\mathscr{Q}^{c}$ is ( $a$ subbase of ) a filter that meets $A$. 
We say that a filter $\mathscr{F}$ is overcoverable with respect to a set $A$ if for every overcover of $A$ there is a finite subfamily that covers an element of $\mathscr{F}$ (in other words, there is a finite subfamily a union of which belongs to $\mathscr{F})$.

$\mathscr{F}$ is said to be overcoverable with respect to a family $\mathscr{H}$ if it is overcoverable with respect to every $H$ in $\mathscr{H}$.

THEOREM 3.7. If $\mathscr{F}$ is $A$-compactoid, then $\mathscr{F}$ is overcoverable with respect to $A$.

Proof. Let $\mathscr{F}$ be $A$-compactoid and consider a family $\mathscr{Q}$ such that for no finite subfamily $Q_{1} \cup Q_{2} \cup \cdots \cup Q_{n} \notin \mathscr{F}$. By Lemma $3.6, \mathscr{Q}^{c}$ is a subbase of a filter that meets $\mathscr{F}$. In view of Proposition 3.1, $A \cap$ Adh $\mathscr{2}^{c}$ $\neq \varnothing$, and by (2.8) we have (3.4). Consequently $\mathscr{Q}$ is not an overcover of $A$.

THEOREM 3.8. If, in a pretopological space, $\mathscr{F}$ is overcoverable with respect to $A$, then $\mathscr{F}$ is $A$-compactoid.

Proof. Let a filter $\mathscr{G}$ meet $\mathscr{F}$. By Lemma 3.6, $\mathscr{G}^{c}$ has no finite subfamily that covers an element of $\mathscr{F}$. Since $\mathscr{F}$ is overcoverable with respect to $A, \mathscr{G}^{c}$ is not an overcover of $A$, hence, in view of Lemma 3.5, $A$ intersects the closure of $\mathscr{G}$ which, in pretopological spaces, is equal to the adherence of $\mathscr{G}$.

Overcovers were called interior covers in [3, p. 285], and a special case (for the filter $\{X\}, X$ being a pretopological space) of Theorems 3.7 and 3.8 was proved [3, p. 783$]$.

We observe that in topological spaces, $\mathscr{F}$ is overcoverable with respect to $A$ if and only if it is coverable with respect to $A$ (for every open cover of $A$ there is a finite subcover of an element of $\mathscr{F}$ ).

COROLlaRY 3.9. A filter $\mathscr{F}$ in a pretopological space is A-compactoid if and only if $\mathscr{F}$ is overcoverable with respect to $A \cap \operatorname{Adh} \mathscr{F}$.

COROLlary 3.10. A subset $A$ of a pretopological space is compactoid if and only if for every overcover of the whole space ( or of $\mathrm{Cl} A$ ) there is a finite subfamily that cover $A$.

COROLlaRY 3.11. A subset $A$ of a pretopological space is compact if and only if its every overcover has a finite subfamily that covers $A$. 
We call a set relatively compact if its closure is compact. It is immediate that

Proposition 3.12. Each relatively compact set is compactoid.

THEOREM 3.13. Let $\pi$ be a pretopology. If a filter $\mathscr{F}$ is $A$-compactoid in $\pi$, then $\mathscr{N}_{\pi}(\mathscr{F})$ is A-compactoid in $\pi^{2}$.

Proof. Let $\mathscr{H}$ meet $\mathscr{N}_{\pi}(\mathscr{F})$. By Corollary 2.2 , for every $H \in \mathscr{H}, \mathrm{Cl}_{\pi} H$ meets $\mathscr{F}$. Therefore the adherence in $\pi$ of the filter generated by $\left\{\mathrm{Cl}_{\pi} H\right.$ : $H \in \mathscr{H}\}$ fulfils

$$
\varnothing \neq A \cap \bigcap_{H \in \mathscr{H}} \mathrm{Cl}_{\pi}\left(\mathrm{Cl}_{\pi} H\right)=A \cap \bigcap_{H \in \mathscr{H}} \mathrm{Cl}_{\pi^{2}} H .
$$

Recall now that $\mathrm{Adh}^{\pi^{2}} \mathscr{H}=\bigcap_{H \in \mathscr{H}} \mathrm{Cl}_{\pi^{2}} H$.

THEOREM 3.14. Let $\mathscr{F}$ be a filter in a pretopological space $(X, \pi)$. If $\mathscr{F}$ is $\mathscr{N}_{\pi}(A)$-compactoid in $\pi$, then $\mathscr{F}$ is $A$-compactoid in $\pi^{2}$.

Proof. Let $\mathscr{U}$ be an ultrafilter finer than $\mathscr{F}$. Since $\mathscr{F}$ is compactoid with respect to $\mathscr{N}_{\pi}(A), \operatorname{Lim}^{\pi} \mathscr{U} \in \mathscr{N}_{\pi}(A)^{\#}$. By virtue of Lemma $2.1, \mathrm{Cl}_{\pi} \operatorname{Lim}^{\pi} \mathscr{U}$ intersects $A$. Since $\mathscr{U}$ is an ultrafilter in a pretopological space, $\operatorname{Lim}^{\pi} \mathscr{U}=$ $\bigcap_{U \in \mathscr{U}} \mathrm{Cl}_{\pi} U$ and thus

$$
\varnothing \neq A \cap \mathrm{Cl}_{\pi}\left(\operatorname{Lim}^{\pi} \mathscr{U}\right) \subset A \cap \bigcap_{U \in \mathscr{U}} \mathrm{Cl}_{\pi^{2}} U,
$$

that is, $\operatorname{Lim}^{\pi^{2}} \mathscr{U}$ intersects $A$.

CoRollary 3.15. Let $\pi$ be a pretopology. If $\mathscr{F}$ is compact in $\pi$ then $\mathscr{N}_{\pi}(\mathscr{F})$ is compact in $\pi^{2}$. If $\mathscr{N}_{\pi}(\mathscr{F})$ is compact in $\pi$, then $\mathscr{F}$ is compact in $\pi^{2}$.

Therefore, in topological spaces (which are equal to their squares) $\mathscr{F}$ is $A$-compactoid if and only if $\mathscr{N}(\mathscr{F})$ is $A$-compactoid, and $\mathscr{F}$ is compact if and only if $\mathscr{N}(\mathscr{F})$ is compact. A practical consequence of this fact, in view of Corollary 2.2, is

COROLlaRY 3.16. In topological spaces a filter $\mathscr{F}$ is compactoid with respect to a set $A$ if and only if for every filter $\mathscr{G}$ which has a base composed of closed sets and meets $\mathscr{F}$, Adh $\mathscr{G} \cap A \neq \varnothing$.

Filters with bases of closed sets will be encountered in the next section. 
THEOREM 3.17. Let $X$ be a pretopological space in which for every ultrafilter $\mathscr{U}, \operatorname{Lim} \mathscr{U}$ is closed (in particular if $X$ is a Hausdorff space). If $\mathscr{N}^{2}(x)$ is compact for each $x \in X$ then the space is topological. ${ }^{2}$

Proof. It is enough to show that $\mathscr{N}^{2}(x) \supset \mathscr{N}(x)$. Let $\mathscr{U}$ be an ultrafilter finer than $\mathscr{N}^{2}(x)$. Thus, by our assumption, $\operatorname{Lim} \mathscr{U} \in \mathscr{N}^{2}(x)^{\#}$ and, by Lemma 2.1, $\mathrm{Cl} \operatorname{Lim} \mathscr{U}=\operatorname{Lim} \mathscr{U} \in \mathscr{N}(x)^{\#}$, and this means $x \in$ Cl $\operatorname{Lim} \mathscr{U}=\operatorname{Lim} \mathscr{U}$ or, equivalently, $\mathscr{U} \supset \mathscr{N}(x)$. Since every filter is equal to the intersection of its ultrafilters, $\mathscr{N}^{2}(x) \supset \mathscr{N}(x)$.

We say that a filter $\mathscr{F}$ semiconverges to a set $A$ if $\mathscr{F} \supset \mathscr{N}(A)$ (semiconverges to a family $\mathscr{A}$ if $\mathscr{F} \supset \mathscr{N}(\mathscr{A})$ ). Previously we called a filter $\mathscr{F}$ finer than $\mathscr{N}(A)$ "adherent to $A$ " [9].

Proposition 3.18. If $\mathscr{F}$ is compactoid it semiconverges to Adh $\mathscr{F}$.

Proof. $Q \in \mathscr{N}(A)$ if and only if $A \subset \operatorname{Int} Q$, so $\{Q\}$ is an overcover of $A$. By Proposition 3.1, $\mathscr{F}$ is Adh $\mathscr{F}$-compactoid and, by Theorem 3.7, $\mathscr{F}$ is overcoverable with respect to Adh $\mathscr{F}$. By setting $A=$ Adh $\mathscr{F}$, we infer that $Q \in \mathscr{F}$.

4. Subregular and regular filters. A filter $\mathscr{F}$ in a pseudotopological space is called subregular if its closure filter $\overline{\mathscr{F}}$ (i.e. the filter generated by $\{\mathrm{Cl} F: F \in \mathscr{F}\})$ is compactoid.

Every subregular filter is compactoid.

A filter $\mathscr{F}$ is said to be regular if $\mathscr{F}=\overline{\mathscr{F}}$. In topological spaces this definition is due to Penot [20], but the concept was known before in the context of open filters (e.g. [21]).

PROPOSITION 4.1. Every regular compactoid filter is subregular and compact.

Let $X$ be a compact nonregular space (e.g. the natural numbers with cofinite sets being an open base). Neighborhood filters in $X$ are subregular but not necessarily regular.

One observes that every filter finer than a subregular filter is subregular; a finite intersection of filters is subregular if and only if all the filters are subregular. A finite intersection of regular filters is regular.

\footnotetext{
${ }^{2}$ THEOREM. If, for every $x \in X, \mathcal{N}(x)$ is compact and, for every ultrafilter $\mathscr{U}, \operatorname{Lim} \mathscr{U}$ is closed, then $X$ is pretopological.
} 
Let $\left\{\mathscr{F}_{i}\right\}_{i \in I}$ be a collection of regular filters which are coarser than a given filter $\mathscr{G}$. Then the supremum $\mathrm{V}_{i \in I} \mathscr{F}_{i}$ is regular.

THEOREM 4.2. Let $\mathscr{F}$ be a subregular filter. Then $\mathrm{Cl} \mathscr{F}$ is compactoid and nonempty; hence Adh $\mathscr{F}$ is compactoid.

Proof. Let $\mathscr{G}$ meet $\mathrm{Cl} \mathscr{F}$, where $\mathscr{F}$ is subregular. By (2.7), $\mathscr{G}$ meets $\overline{\mathscr{F}}$, thus Adh $\mathscr{G} \neq \varnothing$ because $\overline{\mathscr{F}}$ is compactoid. Consequently, $\mathrm{Cl} \mathscr{F}$ is compactoid.

A result of [20, Prop. 14] follows from

COROLLARY 4.3. The pretopological adherence of every regular compactoid filter is compactoid. The adherence of a regular compactoid filter in a topological space is compact and closed.

The pretopological adherence and adherence in the above statements are, of course, nonempty.

A pseudotopological space is called subregular if every convergent filter of it is subregular. Therefore a pretopological space is subregular if and only if every neighborhood filter of it is subregular. Observe that every compact space is subregular. A subspace of a subregular space need not be subregular.

THEOREM 4.4. The following properties are equivalent:

(i) $A$ space is subregular.

(ii) Every convergent ultrafilter is subregular.

(iii) Every compactoid filter is subregular.

Proof. (i) $\Rightarrow$ (ii) is obvious. (ii) $\Rightarrow$ (iii). Let $\mathscr{F}$ be a compactoid filter and $\mathscr{U}$ an ultrafilter finer than $\overline{\mathscr{F}}$. By Corollary $2.2, \mathscr{N}(\mathscr{U})$ meets $\mathscr{F}$. An ultrafilter $\mathscr{V}$ finer than $\mathscr{N}(\mathscr{U}) \vee \mathscr{F}$ is convergent because $\mathscr{F}$ is compactoid. On the other hand, $\mathscr{V}$ meets $\mathscr{N}(\mathscr{U})$ and, by Corollary $2.2, \mathscr{U}$ meets $\overline{\mathscr{V}}$, thus $\mathscr{U} \supset \bar{V}$. By (ii), $\overline{\mathscr{V}}$ is compactoid, hence $\operatorname{Lim} \mathscr{U} \neq \varnothing$, which proves the subregularity of $\mathscr{F}$. (iii) $\Rightarrow$ (i). Every convergent filter is compactoid.

As a consequence of Theorems 4.2 and 4.4, we have

COROLLARY 4.5. The pretopological adherence of every compactoid filter in a subregular space is compactoid.

COROLlaRY 4.6. The closure of each compactoid subset of a subregular space is compactoid. 
We complement now Proposition 3.12 by

COROLlARY 4.7. Each compactoid subset of a subregular topological space is relatively compact.

Proof. Let $A$ be a compactoid set; then $\mathscr{N}_{\iota}(A)$ is compactoid and, by subregularity, $\mathrm{Cl} A$ is (closed and) compactoid, hence compact.

We conclude that in subregular topological spaces, compactoid and relatively compact sets coincide.

In subregular spaces we have a reinforcement of Proposition 3.18 that generalizes [20, Thm 15]

Proposition 4.8. A compactoid filter in a subregular pretopological space semiconverges to a compactoid set. If a filter in a topological space semiconverges to a compactoid set, then it is compactoid.

There are examples that the second part of the above proposition need not be true in pretopological spaces.

A pseudotopological space is called regular if for every filter $\mathscr{F}$, $\operatorname{Lim} \mathscr{F}=\operatorname{Lim} \overline{\mathscr{F}}$. Accordingly a pretopological space is regular if and only if its every neighborhood filter is regular. A subspace of a regular space is regular.

Our definition does not require that the space be Hausdorff ( or $T_{1}$ ).

It is immediate that

Proposition 4.9. Every regular space is subregular.

An example of a subregular space which is neither regular nor compact is given by the topological infinite sum of copies of the space of natural numbers with the cofinite topology (open sets are the complements of finite sets).

THEOREM 4.10. For a pretopological space $X$ the following are equivalent:

(i) $X$ is regular.

(ii) For every $A \subset X$ and $x \notin \mathrm{Cl} A, \mathscr{N}(A)$ and $\mathscr{N}(x)$ are disjoint.

(iii) $\mathrm{Cl} \mathscr{N}(A)=\mathrm{Cl} A$ for each $A \subset X$.

(iv) $\mathrm{Cl} \mathscr{N}(\mathscr{A})=\mathrm{Cl} \mathscr{A}$ for each $\mathscr{A} \subset 2^{X}$.

(v) For each $A \subset X$ and each filter $\mathscr{F}$ semiconvergent to $A$, Adh $\mathscr{F}=$ $\mathrm{Cl} \mathscr{F} \subset \mathrm{Cl} A$. 
Proof. (i) $\Rightarrow$ (ii) Let $x \notin \mathrm{Cl} A$. By (2.10), $A^{c} \in \mathscr{N}(x)$ and, in view of regularity, there is $M \in \mathscr{N}(x)$ such that $\mathrm{Cl} M \subset A^{c}$. In other words, $A \subset$ Int $M^{c}$ or, equivalently, $M^{c} \in \mathscr{N}(A)$. (ii) $\Rightarrow$ (iii). We always have $\mathrm{Cl} A \subset \mathrm{Cl} \mathscr{N}(A)$. Assume (ii) and let $x \notin \mathrm{Cl} A$; then $\mathscr{N}(x)$ and $\mathscr{N}(A)$ are disjoint (there is $Q \in \mathscr{N}(A)$ such that $Q^{c} \in \mathscr{N}(x)$ and, by (2.10), $x \notin \mathrm{Cl} Q$ ), thus $x \notin \mathrm{Cl} \mathscr{N}(A)$. (iii) $\Rightarrow$ (iv) In view of (2.11) and (2.7), $\mathrm{Cl} \mathscr{N}(\mathscr{A})=\bigcap_{A \in \mathscr{A}} \mathrm{Cl} \mathscr{N}(A)$, which, by (iii), is equal to $\mathrm{Cl} \mathscr{A}$. (iv) $\Rightarrow$ (v) If $\mathscr{F} \supset \mathscr{N}(A)$ then $\mathrm{Cl} \mathscr{F} \subset \mathrm{Cl} \mathscr{N}(A)=\mathrm{Cl}(A)$ by (iv). (v) $\Rightarrow$ (iii) We have $\mathscr{N}_{\iota}(A) \supset \mathscr{N}(A)$; thus $\mathrm{Cl} A=\mathrm{Cl} \mathscr{N}_{\iota}(A) \subset \mathrm{Cl} \mathscr{N}(A) \subset \mathrm{Cl} A$. (iii) $\Rightarrow$ (ii) Let $x \notin \mathrm{Cl} A$, thus, by (iii), $x \notin \mathrm{Cl} \mathscr{N}(A)$, that is, $\mathscr{N}(x)$ and $\mathscr{N}(A)$ are disjoint. (ii) $\Rightarrow$ (i) Let $Q \in \mathscr{N}(x)$, i.e., $x \notin \mathrm{Cl} Q^{c}$. By (ii) there is $M \in$ $\mathscr{N}(x)$ such that $M^{c} \in \mathscr{N}\left(Q^{c}\right)$, hence $Q^{c} \subset$ Int $M^{c}$ or, equivalently, $\mathrm{Cl} M$ $\subset Q$, proving that $Q \in \bar{N}(x)$.

The implication (i) $\Rightarrow$ (v) entails [9, Thm 5].

As in (v) of Theorem 4.10, in the case of Hausdorff topological spaces, Adh $\mathscr{F} \subset A$ whenever $\mathscr{F}$ is semiconvergent to $A$ and $A$ is a compact set ([20; Proposition 13, a]). However, unlike (v), this need not be true for pretopological spaces.

ExAmple 4.11. Let $X=\mathbf{R}, A=\{-1 / n: n \in \mathbf{N}\} \cup\{0\}$ and let $\left\{\mathscr{U}_{n}\right\}_{n=0}^{\infty}$ be a collection of free ultrafilters such that $(n+1, n+2) \in \mathscr{U}_{n}$ for all $n$. Take a free ultrafilter $\mathscr{U}_{\infty} \supset\left(\cap_{n \geq 0} \mathscr{U}_{n}\right) \vee \mathscr{N}_{\iota}(\{(n+1,+\infty)$ : $n \geq 0\})$ and define

$$
\mathscr{N}_{\pi}(x)= \begin{cases}\mathscr{N}_{\tau}\left(-\frac{1}{n}\right) \cap \mathscr{U}_{n} & \text { if } x=-\frac{1}{n}, n=1,2, \ldots, \\ \mathscr{N}_{\tau}(0) \cap \mathscr{U}_{0} & \text { if } x=0, \\ \mathscr{N}_{\iota}(x) \cap \mathscr{U}_{\infty} & \text { if } x \notin A,\end{cases}
$$

where $\tau$ denotes the standard topology of $\mathbf{R}$. Observe that $\pi$ is a Hausdorff pretopology, $A$ is compact and $\mathscr{N}_{\pi}(A)$ is semiconvergent to $A$ but $A^{c} \cap$ $\operatorname{Adh}^{\pi} \mathscr{N}_{\pi}(A) \neq \varnothing$.

THEOREM 4.12. If a filter $\mathscr{F}$ in a regular pretopological space is A-compactoid, then Adh $\mathscr{F}$ is A-compactoid.

Proof. We show first that the assumption implies that $\overline{\mathscr{F}}$ is $A$-compactoid. Let $\mathscr{G}$ be a filter that meets $\overline{\mathscr{F}}$. By Corollary $2.2, \mathscr{N}(\mathscr{G})$ meets $\mathscr{F}$ and thus Adh $\mathscr{N}(\mathscr{G}) \cap A \neq \varnothing$. By Theorem 4.10, Adh $\mathscr{G} \cap A \neq \varnothing$. If now a filter $\mathscr{G}$ meets Adh $\mathscr{F}$, then it meets $\overline{\mathscr{F}}$, hence, by the first part of the proof, $\operatorname{Adh} \mathscr{G} \cap A \neq \varnothing$. 
This Theorem generalizes [9, Thm 1] [20, Prop. 14].

COROLLARY 4.13. The adherence of a compactoid filter in a regular pretopological space is compact. In particular, a compactoid set in a regular pretopological space is relatively compact. ${ }^{3}$

COROLlaRY 4.14. A filter in a regular topological space is $\mathscr{H}$-compactoid if and only if it semiconverges to an $\mathscr{H}$-compactoid set.

A pretopological space is called completely Hausdorff if for all elements $x \neq y$, the filters $\overline{\mathcal{N}(x)}$ and $\mathscr{N}(y)$ are disjoint. Clearly every Hausdorff regular pretopological space is completely Hausdorff (and subregular). The converse is also valid.

THEOREM 4.15. Every completely Hausdorff subregular pretopological space is regular.

Proof. Let $\mathscr{U} \supset \overline{\mathscr{N}(x)}$. Since $\overline{\mathcal{N}(x)}$ is compactoid, Lim $\mathscr{U} \neq \varnothing$. If for some $y \neq x, y$ were in $\operatorname{Lim} \mathscr{U}$, then $\mathscr{U}$ would be finer than $\mathscr{N}(y)$, which is impossible, because the space is completely Hausdorff. Thus $\mathscr{N}(x) \subset \mathscr{U}$. Taking the intersection of all ultrafilters of $\overline{\mathcal{N}(x)}$, we get $\mathcal{N}(x) \subset \overline{\mathscr{N}(x)}$, proving regularity.

THEOREM 4.16. Every subregular Hausdorff topological space is regular.

Proof. Since the space is topological, Adh $\overline{\mathscr{N}(x)}=\operatorname{Adh} \mathscr{N}(x)$, which is equal to $\{x\}$ because the space is Hausdorff. By subregularity $\overline{\mathscr{N}(x)}$ is compactoid, and in view of Proposition $3.18 \overline{\mathcal{N}(x)} \supset \mathscr{N}($ Adh $\mathscr{N}(x))=$ $\mathscr{N}(x)$.

We observe that

Proposition 4.17. A product of pseudotopological spaces is subregular if and only if every space is subregular.

This is a simple consequence of Theorem 5.1 and Corollary 5.8 of the next section.

\footnotetext{
${ }^{3}$ It follows from Corollary 4.13 that a Hausdorff regular compact pretopology is a topology. On the other hand, a Hausdorff regular compact pseudotopology is a pretopology thus a topology. The above is also a result of Theorem 1 of G. D. Richardson and D. C. Kent, Regular compactifications on convergence spaces, Proc. Amer. Math. Soc., 31 (1972), 571-573.
} 
Finally, the set of regular topologies with the usual order is inductive, but the set of subregular topologies is not.

EXAMPLE 4.18. Let $\mathscr{F}$ be the cocountable filter in $\mathbf{R}$ (i.e. the filter composed of the complements of countable sets). Define the following sequence of topologies $\left\{\tau_{n}\right\}$ by giving their neighborhood filters

$$
\mathscr{N}_{n}(x)= \begin{cases}\mathscr{F} \cap \mathscr{N}_{\iota}(x) & \text { for } x<n, \\ \{\mathbf{R}\} & \text { for } x \geq n .\end{cases}
$$

These topologies are subregular because they are compact. They form a chain and their supremum is the topology with the neighborhood filters

$$
\mathscr{N}(x)=\mathscr{F} \cap \mathscr{N}_{\iota}(x)
$$

which is not subregular: $\overline{\mathscr{N}(x)}=\{\mathbf{R}\}$ and $\mathbf{R}$ is not compact.

5. Tychonoff theorem and subcontinuous relations. Let $\left\{X_{\imath}, \theta_{i}\right\}_{i \in I}$ be a collection of pseudotopological spaces. The product pseudotopology $\theta=\prod_{i \in I} \theta_{i}$ is the coarsest pseudotopology on $X=\prod_{i \in I} X_{i}$ for which all projection mappings $p_{i}$ are continuous. Accordingly, for each filter $\mathscr{F}$ in $X$

$$
x \in \operatorname{Lim}^{\theta} \mathscr{F} \text { iff } p_{i}(x) \in \operatorname{Lim}^{\theta_{i}} p_{i}(\mathscr{F}) \quad \text { for each } i \in I .
$$

THEOREM 5.1. Let $\mathscr{F}$ be a filter in the product space $X$ and let $H=\prod_{i \in I} H_{l}$. If for every $i \in I, p_{i}(\mathscr{F})$ is $H_{i}$-compactoid, then $\mathscr{F}$ is $H$-compactoid.

Proof. Let $\mathscr{U}$ be an ultrafilter of $\mathscr{F}$. Then for each $i \in I, p_{l}(\mathscr{U})$ is an ultrafilter finer than $p_{i}(\mathscr{F})$, hence there is $x_{i}$ in $\operatorname{Lim}^{\theta_{t}} p_{i}(\mathscr{U}) \cap H_{i}$ and, by (5.1), $x=\prod_{i \in I} x_{i}$ belonts to $\operatorname{Lim}^{\theta} \mathscr{U} \cap H$.

Accordingly, a filter is compactoid, if its every projection is compactoid. However, this is not in general true about compact filters.

THEOREM 5.2. Let $\mathscr{F}_{i}$ be a compact filter in $X_{i}$ for $i \in I$. Then $\prod_{i \in I} \mathscr{F}_{l}$ is compact.

Proof. Let $F \in \Pi_{i \in I} \mathscr{F}_{i}$, thus $F$ includes a set of the form $\Pi_{\imath \in I} F_{i}$, where $F_{i} \in \mathscr{F}_{i}$. Since $p_{j}\left(\Pi_{i \in I} \mathscr{F}_{i}\right)=\mathscr{F}_{j}$ is compactoid with respect to $p_{j}(F) \supset F_{j}$ for every $j \in I, \prod_{i \in I} \mathscr{F}_{l}$ is compactoid with respect to $F$. As $F$ was an arbitrary element of the product filter, the latter is compact.

COROLlaRY 5.3. (Tychonoff theorem.) The product of topological spaces is compact if every of those spaces is compact. 
Let $X$ and $Y$ be pseudotopological spaces. A relation $\Gamma: X \rightrightarrows Y$ is called subcontinuous at $x$ if for every ultrafilter $\mathscr{U}$ such that $x \in \operatorname{Lim} \mathscr{U}$, the filter $\Gamma \mathscr{U}$ is compactoid. $\Gamma$ is subcontinuous if it is subcontinuous at every $x$ of the closure of $\Gamma^{-1} Y$. If $X$ is a pretopological space, then $\Gamma: X \rightrightarrows Y$ is subcontinuous at $x$ whenever $\Gamma \mathscr{N}(x)$ is compactoid.

Since every convergent filter is compactoid, every continuous mapping is a subcontinuous relation. If $Y$ is a compact space, then for every $X$ and every $\Gamma: X \rightrightarrows Y, \Gamma$ is subcontinuous. A finite union and an arbitrary product of subcontinuous (at $x$ ) relations are subcontinuous (at $x$ ). A subrelation of a subcontinuous relation is subcontinuous.

The original definitions of subcontinuous relations [14] [23] were expressed in terms of nets in topological spaces. We shall evoke them by

Proposition 5.4. Let $X$ and $Y$ be topological spaces. $A$ relation $\Gamma$ : $X \rightrightarrows Y$ is subcontinuous at $x$ if and only if for every net $\left\{x_{\alpha}\right\}_{\alpha \in A}$ convergent to $x$ and every net $\left\{y_{\alpha}\right\}_{\alpha \in A}$ such that $y_{\alpha} \in \Gamma_{x_{\alpha}}$ there is a convergent subnet of $\left\{y_{\alpha}\right\}_{\alpha \in A}$.

Proof. Suppose that $\Gamma$ is subcontinuous at $x$. Then the section filter of $\left\{y_{\alpha}\right\}_{\alpha \in A}$ is finer than $\Gamma\left\{x_{\alpha}\right\}_{\alpha \in A}$ (the image of the section filter of $\left.\left\{x_{\alpha}\right\}_{\alpha \in A}\right)$ and thus is finer than $\Gamma \mathscr{N}(x)$. Thus $\left\{y_{\alpha}\right\}_{\alpha \in A}$ has nonvoid adherence (admits a cluster point) so it has a convergent subnet.

Vice versa, let $\mathscr{G}$ meet $\Gamma \mathcal{N}(x)$. Then for every $G \in \mathscr{G}$ and $Q \in \mathscr{N}(x)$ there is $y_{G, Q} \in G \cap \Gamma Q$ and thus there is $x_{G, Q} \in \Gamma^{-1} y_{G, Q} \cap Q$. The net $\left\{x_{G, Q}\right\}_{(G, Q) \in \mathscr{G} \times \mathcal{N}(x)}$ (usually ordered) converges to $x$, so that there is a convergent subnet such that its section filter is finer than $\mathscr{G}$. Consequently, Adh $\mathscr{G} \neq \varnothing$.

THEOREM 5.5. A relation $\Gamma: X \rightrightarrows Y$ is subcontinuous if and only if for every compactoid filter $\mathscr{F}$ (that meets $\Gamma^{-1} Y$ ), $\Gamma \mathscr{F}$ is a compactoid filter.

Proof. The sufficiency is obvious. Let $\mathscr{F}$ be compactoid and let $\mathscr{G}$ be a filter that meets $\Gamma \mathscr{F}$. Equivalently, $\Gamma^{-1} \mathscr{G}$ meets $\mathscr{F}$ and thus for each ultrafilter $\mathscr{U}$ finer than $\Gamma^{-1} \mathscr{G} \vee \mathscr{F}, \operatorname{Lim} \mathscr{U}$ is nonempty. In particular, $\mathscr{U}$ meets $\Gamma^{-1} \mathscr{G}$, thus $\Gamma \mathscr{U}$ meets $\mathscr{G}$ and, moreover, $\Gamma \mathscr{U} \vee \mathscr{G} \supset \Gamma \mathscr{U}$. We conclude that

$$
\varnothing \neq \operatorname{Adh}(\Gamma \mathscr{U} \vee \mathscr{G}) \subset \operatorname{Adh} \mathscr{G},
$$

which proves that $\Gamma \mathscr{F}$ is a compactoid filter.

It is rather exciting that Theorem 4.4 is a corollary of Theorem 5.5; this fact will be discussed in a future paper. 
COROLlARY 5.6. The image of every compactoid set by a subcontinuous relation is compactoid.

On recalling Proposition 3.12 and Corollary 4.7, we obtain a reinforcement of [23, Thm. 2.1].

COROllary 5.7. Let $\Gamma$ be a subcontinuous relation from a pseudotopological space to a subregular topological space. If $K$ is relatively compact, then $\Gamma K$ is relatively compact.

For projections one gets stronger results than those for general subcontinuous relations, in particular, generalizing the converse of the Tychonoff theorem.

THEOREM 5.8. Let $H$ be a subset of a product pseudotopological space $\Pi_{i \in I} X_{\imath}$ and $\mathscr{F}$ a filter therein. If $\mathscr{F}$ is $H$-compactoid, then for each $i \in I$, $p_{i}(\mathscr{F})$ is $p_{i}(H)$-compactoid.

Proof. Let $\mathscr{G}$ meet $p_{i}(\mathscr{F})$. Let $\mathscr{U}$ be an ultrafilter of $p_{i}^{-1}(\mathscr{G}) \vee \mathscr{F}$. By assumption, $H \cap \operatorname{Lim} \mathscr{U} \neq \varnothing$, thus $p_{i}(\operatorname{Lim} \mathscr{U}) \cap p_{i}(H) \neq \varnothing$. On the other hand, $p_{i}(\operatorname{Lim} \mathscr{U}) \subset \operatorname{Lim}^{i} p_{i}(\mathscr{U}) \subset \operatorname{Adh}^{i} \mathscr{G}$.

COROLlaRY 5.9. Every projection of a compact filter is compact.

A relation is compact-to-compact if it maps every compact set into a compact set. Similarly a relation is compact-to-closed if the image of every compact set is closed.

Let $A$ be a nonempty subset of a pseudological space. The cocompact filter of $A, X(A)$ is the filter for which

$$
\left\{K^{c}: K \cap A=\varnothing, K \text { compact }\right\}
$$

constitutes a base. A filter $\mathscr{F}$ cocompactly semiconverges to $A$ provided that $\mathscr{F} \supset X_{(}(A)$. Consequently,

$\mathscr{F}$ cocompactly semiconverges to $A$ if and only if for each compact set $K$ in $\mathscr{F} \#, K \cap A \neq \varnothing$.

Proposition 5.10.

(i) If in a pretopological space Adh $\mathscr{F} \subset A$, then $\mathscr{F}$ cocompactly semiconverges to $A$.

(ii) If a space is hereditary locally compact ( see §8) and $\mathscr{F}$ is cocompactly semiconvergent to a closed set $A$, then Adh $\mathscr{F} \subset A$. 
Proof. (i) Let $K$ be a compact set disjoint from $A$ and let $x \in K$. Hence $\mathscr{N}(x)$ and $\mathscr{F}$ are disjoint: there is $Q_{x}$ such that $x \in \operatorname{Int} Q_{x}$ and $Q_{x}^{c} \in \mathscr{F}$. The collection $\left\{Q_{x}\right\}_{x \in K}$ of so chosen sets is an overcover of $K$ thus there is a finite cover $Q_{1}, \ldots, Q_{n}$ of $K$ by elements of that collection. The element $Q_{1}^{c} \cap \cdots \cap Q_{n}^{c}$ of $\mathscr{F}$ is thus disjoint from $K$ and $\mathscr{F} \supset \backslash(A)$.

(ii) Let $x \notin A$. Since $A$ is closed there is a compact neighborhood $K$ of $x$ disjoint from $A$. By assumption, $\mathscr{F}$ is disjoint from $K$, hence $x \notin$ Adh $\mathscr{F}$.

The assumption of local compactness in (ii) is essential. Indeed, the above considerations relate to the topologizability problems for upper limits (see e.g. [1] [5]) and cocompact semiconvergence amounts to the convergence in the compact-open topology.

Let $\Gamma: Y \rightrightarrows X$ be a relation in pretopological spaces. Call $\Gamma$ cocompactly stable at $y$ if $\Gamma \mathcal{N}(y) \supset X(\Gamma y)$, and cocompactly stable if the above holds for each $y$ (equivalently, for each $y \in \mathrm{Cl}^{-1} X$ ).

Using a duality argument we establish

Proposition 5.11. A relation $\Gamma$ is cocompactly stable at $y$ if and only if for every compact subset $K$ of $X, y \in \mathrm{Cl} \Gamma^{-1} K$ implies that $y \in \Gamma^{-1} K$.

Corollary 5.12. A relation is compact-to-closed if and only if its inverse is cocompactly stable.

COROLlaRY 5.13. Let $\Gamma$ be a a relation from a pretopological space to a subregular topological space. If $\Gamma$ is subcontinuous and $\Gamma^{-1}$ is cocompactly stable, then $\Gamma$ is compact-to-compact.

A relation $\Gamma: Y \rightrightarrows X$ (from a pretopological space to another) is graph-closed at $y$ if Adh $\Gamma \mathscr{N}(y) \subset \Gamma y . \Gamma$ is (everywhere) graph-closed if and only if $\Gamma$ is a closed subset of $Y \times X$. A relation is graph-closed if and only if its inverse is graph-closed. By Proposition 5.10 every graph-closed (at $y$ ) relation is cocompactly stable (at $y$ ) and the converse holds if $X$ locally compact and the relation has closed values. These remarks and Corollary 5.13 entail [23, Prop. 3.2].

A relation $\Gamma: X \rightrightarrows Y$ is pretopological spaces is called upper semicontinuous at $x$ if $\Gamma \mathscr{N}(x)$ semiconverges to $\Gamma x$. This notion coincides with the classical concepts of upper semicontinuity in topological spaces ([5] [15]).

In view of Proposition 4.8 every subcontinuous graph-closed relation is upper semicontinuous [23, Thm. 3.1]. Theorem $4.10((\mathrm{i}) \Leftrightarrow(\mathrm{v}))$ entails that every closed-valued upper semicontinuous relation into a space $Y$ is graph-closed if and only if $Y$ is regular (extension of a classical result of 
Kuratowski). In $§ 7$ we explore subtler interdependences between upper semicontinuity and subcontinuity.

6. Countably compactoid and pseudocompactoid filters. A filter $\mathscr{F}$ in a pseudotopological space $X$ is called countably compactoid with respect to a set $H$ (countably $H$-compactoid) if for every countably based filter $\mathscr{G}$ that meets $\mathscr{F}, H \cap \operatorname{Adh} \mathscr{G} \neq \varnothing$. It is called countably compactoid if it is countably $X$-compactoid, and countably $\mathscr{H}$-compactoid for a family $\mathscr{H} \subset 2^{X}$ if it is countably $H$-compactoid for every $H \in \mathscr{H}$. Finally, $\mathscr{F}$ is called countably compact if it is countably $\mathscr{F}$-compactoid.

Every $\mathscr{H}$-compactoid filter is countably $\mathscr{H}$-compactoid.

A filter is called countably overcoverable with respect to $\mathscr{H}$ if for every $H \in \mathscr{H}$ and every countable overcover $\mathscr{Q}$ of $H$ there is a finite subfamily of $\mathscr{Q}$ that covers an element of $\mathscr{F}$.

Using Lemmas 3.5 and 3.6, as we have done in connection with compactoid filters, we get

THEOREM 6.1. If $\mathscr{F}$ is countably A-compactoid, then $\mathscr{F}$ is countably overcoverable with respect to $A$.

In pretopological spaces the converse is true.

Several facts about countably compactoid filters are analogous to those concerning compactoid filters. It seems not very interesting to write them down systematically. Let us mention that a subset of a pseudotopological space is countably compactoid (compact) if and only if its discrete filter is countably compactoid (compact). Some results analogous to those of $\$ 4$ may be obtained by introducing the notion of countably subregular filters which are those filters the closure filters of which are countably compactoid.

THEOREM 6.2. The pretopological adherence of every countably subregular filter is countably compactoid.

THEOREM 6.3. Every regular countably compactoid filter is countably subregular and countably compact.

What is more intriguing about countably compactoid filters is where the analogy with compactoid filters fails.

Already for two countably compact topological spaces $X, Y$ the product $X \times Y$ need not be countably compact ([11, 3.10.19]).

We shall see that there is no analogue of Proposition 3.1. 
A filter $\mathscr{F}$ is called countably quasi $A$-compactoid if for every countably based filter $\mathscr{M}$ finer than $\mathscr{F}, A \cap \operatorname{Adh} \mathscr{M} \neq \varnothing$.

Proposition 6.4. A filter $\mathscr{F}$ is countably quasi $A$-compactoid if and only if for every elementary filter $\mathscr{E}$ finer than $\mathscr{F}, A \cap \operatorname{Adh} \mathscr{E} \neq \varnothing$.

Countably compactoid filters are countably quasi compactoid. For countably based filters the two notions coincide. In particular, they coincide for discrete filters, hence for sets and spaces. One notes that a countably based filter $\mathscr{F}$ is countably compactoid (or, equivalently, countably quasi compactoid) if and only if it is countably Adh $\mathscr{F}$-compactoid.

In general, countable and countable quasi compactoidness differ.

EXAMPLE 6.5. Let $(\mathbf{R}, \iota)$ be the space of reals with the discrete topology. Consider the cocountable filter $\mathscr{C}$ in $\mathbf{R}$, that is, the filter composed of those sets the complements of which are countable. $\mathscr{C}$ is countably quasi compactoid, since no elementary filter is finer than $\mathscr{C} . \mathscr{C}$ is not countably compactoid, because the countably based filter generated by $\left\{\left(-\frac{1}{n}, \frac{1}{n}\right) \backslash\{0\}\right\}_{n \in \mathrm{N}}$ meets $\mathscr{C}$ but its adherence is empty.

An extended-real-valued function $f$ on a pseudotopological space is lower semicontinuous at $x$ if for every filter $\mathscr{F}$ with $x \in \operatorname{Lim} \mathscr{F}$

$$
\operatorname{li}_{\mathscr{F}} f=\sup _{F \in \mathscr{F}} \inf _{x^{\prime} \in F} f\left(x^{\prime}\right) \geq f(x)
$$

The epitopology of $f$ (epi $f$-topology), $\tau($ epi $f$ ) is defined as the coarsest topology on $X$ for which $f$ is lower semicontinuous (everywhere). The open sets of the epitopology of $f$ are precisely

$$
\{x: f(x)>r\}, \quad r \in \mathbf{R},
$$

the empty set and the whole space.

Notice that for a filter $\mathscr{F},(6.1)$ holds if and only if $\mathscr{F}$ converges to $x$ in the epitopology of $f$. Consequently, a function $f$ is lower semicontinuous for a pseudotopology $\theta$ if and only if $\tau($ epi $f) \subset \theta$. Therefore, the coarsest pseudotopology for which all lower $\theta$-semicontinuous functions are lower semicontinuous functions is a topology (coarser than the associated topology of $\theta$ ).

A filter $\mathscr{G}$ is said pseudocompactoid if and only if for each lower semicontinuous function $f: X \rightarrow \mathbf{R} \cup\{+\infty\}$,

$$
\operatorname{li}_{\mathscr{F}} f=\sup _{G \in \mathscr{G}} \inf _{x^{\prime} \in G} f\left(x^{\prime}\right)>-\infty \text {. }
$$

LEMMA 6.6. Let $f: X \rightarrow \mathbf{R} \cup\{+\infty\}$. If $\{x: f(x) \leq \inf (f)\} \neq \varnothing$, then each filter is countably compactoid for $\tau$ (epi $f$ ). If $\{x: f(x) \leq \inf (f)\}=\varnothing$, 
then a filter $\mathscr{G}$ is countably compactoid for $\tau($ epi $f)$, if and only if $\operatorname{li}_{\mathscr{G}} f>$ $\inf (f)$.

\section{We infer that}

THEOREM 6.7. Every countably compactoid filter is pseudocompactoid.

A space $X$ is pseudocompact if the filter $\{X\}$ is pseudocompactoid, that is, whenever for every lower semicontinuous function $f: X \rightarrow \mathbf{R} \cup$ $\{\infty\}, \inf _{x \in X} f(x)>-\infty$. In the case of topological pseudocompact spaces this infimum is attained [11, p. 263]. This is not the case of pseudocompactoid filters where neither supremum nor infimum in (6.2) need be attained.

However,

Proposition 6.8. If $\mathscr{G}$ is a countably compactoid filter and $f$ is a lower semicontinuous function, then there is $x_{0} \in \mathrm{Adh} \mathscr{G}_{\text {such }}$ that $f\left(x_{0}\right)=\mathrm{li}_{\mathscr{G}} f$.

Proof. Let $\left\{r_{n}\right\}_{n \in \mathbf{N}}$ be a sequence strictly decreasing to $\mathrm{li}_{\mathscr{G}} f$. The filter generated by $\left\{\left\{x: f(x) \leq r_{n}\right\}\right\}_{n \in \mathbf{N}}$ meets $\mathscr{G}$ and, $\operatorname{Adh}\left\{\left\{x: f(x) \leq r_{n}\right\}\right\}_{n \in \mathbf{N}}$ meets Adh $\mathscr{G}$. Thus $\bigcap_{r_{n} \downarrow \mathrm{li}_{g} f}\left\{x: f(x) \leq r_{n}\right\} \cap \operatorname{Adh} \mathscr{G} \neq \varnothing$, hence $\{x$ : $\left.f(x) \leq \operatorname{li}_{\mathscr{G}} f\right\} \cap \operatorname{Adh} \mathscr{G} \neq \varnothing$.

On the other hand,

THEOREM 6.9. Let $\mathscr{F}$ be a countably compact filter and $f$ a lower semicontinuous function. If there is $F_{0} \in \mathscr{F}$ such that $\inf _{F_{0}} f=\operatorname{li}_{\mathscr{F}} f$, then $f$ attains its infimum on $F_{0}$.

Proof. By assumption, $F_{0} \subset\left\{x: f(x) \geq \operatorname{li}_{\mathscr{F}} f\right\}$. If the infimum were not attained, $F_{0}$ would be a subset of $\left\{x: f(x)>\operatorname{li}_{\mathscr{F}} f\right\}$. Let $\left\{r_{n}\right\}_{n \in \mathbf{N}}$ decrease to $\operatorname{li}_{\mathscr{F}} f$. Then $\left\{\left\{x: f(x)>r_{n}\right\}\right\}_{n \in \mathbf{N}}$ is an overcover of $F_{0}$ and, by countable compactness, there is $n \in \mathbf{N}$ such that $\left\{x: f(x)>r_{n}\right\}$ belongs to $\mathscr{F}$. This is a contradiction.

7. Totally bounded and semiconvergent filters. Let $(X, \mathfrak{H})$ be a uniform space. A filter $\mathscr{F}$ in $X$ is said to be totally bounded if for every $U \in \mathfrak{U}$ there exist a finite set $M \subset X$ and $F \in \mathscr{F}$ such that $F \subset U(M)$. If we consider the topology associated with the uniformity, then

Proposition 7.1. Every compactoid filter is totally bounded. In complete uniform spaces every totally bounded filter is compactoid. 
Proof. The first statement is obvious. Suppose that $\mathscr{F}$ is a totally bounded filter in a complete uniform space. Let $\mathscr{U}$ be an ultrafilter finer than $\mathscr{F}$. By total boundedness for each $U \in \mathfrak{U}$ there is a finite set $M$ such that

$$
\bigcup_{m \in M} U(m)=U(M) \in \mathscr{F} \subset \mathscr{U}
$$

thus for some $m \in M, U(m)$ belongs to the ultrafilter $\mathscr{U}$. Therefore $\mathscr{U}$ contains arbitrary small sets and converges in view of completeness. This proves that $\mathscr{F}$ is a compactoid filter.

THEOREM 7.2. In Dieudonné complete (topological) space every countably based countably compactoid filter is compactoid.

Proof. Let $\mathfrak{U}$ be a complete uniformity of the space and let $\mathscr{F}$ be a countably compactoid filter admitting a base $\left\{F_{n}\right\}_{n \in \mathbf{N}}$. By Proposition 7.1 , it is enough to show that $\mathscr{F}$ is totally bounded. Suppose not: there is $U \in \mathfrak{U}$ such that for every finite set $M$ and each $F \in \mathscr{F}, F \backslash U(M) \neq \varnothing$. Let $x_{1}$ be any element of $F_{1}$. Suppose we have selected a set $\left\{x_{1}, \ldots, x_{n}\right\}$ such that $x_{i} \in F_{l}$ and $\left(x_{l}, x_{k}\right) \notin U$ for $i \neq k$. Put

$$
x_{n+1} \in F_{n+1} \backslash \bigcup_{i=1}^{n} U\left(x_{i}\right) \text {. }
$$

Obviously, $\left(x_{n+1}, x_{\imath}\right) \notin U$ for $i \leq n$. The sequence $\left\{x_{n}\right\}_{n \in \mathbf{N}}$ has no cluster point, which, in view of Proposition 6.4, is a contradiction.

THEOREM 7.3. If a countably based filter $\mathscr{F}$ in a Dieudonné complete space semiconverges to a set from which it is disjoint, then $\mathscr{F}$ is compactoid.

Proof. Let $\mathscr{F}$ be a countably based filter semiconvergent to $A$. In view of $\S 6$ and Theorem 7.2 , it is enough to show that $\mathscr{F}$ is countably quasi compactoid. Suppose that, on the contrary, there is a sequence $\left\{x_{n}\right\}_{n \in \mathbf{N}}$ the elementary filter of which is finer than $\mathscr{F}$ but with empty adherence. Consequently, $\left\{x_{n}\right\}_{n \in \mathbf{N}}$ is a closed set disjoint from $A$ but intersecting every element of $\mathscr{F}$. Consequently, the open set $\left(\left\{x_{n}\right\}_{n \in \mathrm{N}}\right)^{c}$ is not in $\mathscr{F}$, contradicting the semiconvergence of $\mathscr{F}$ to $A$.

We complement Theorem 4.10 by

THEOREM 7.4. Let $X$ be a first countable space. If a countably based filter $\mathscr{F}$ in $X$ semiconverges to a set $A$ from which it is disjoint, then Adh $\mathscr{F} \subset A$. 
Proof. Let $x \in \operatorname{Adh} \mathscr{F} A$. Let $\left\{Q_{n}\right\}_{n \in \mathbf{N}}$ be a base of $\mathscr{N}(x)$ and $\left\{F_{n}\right\}_{n \in N}$ a base of $\mathscr{F}$ of sets disjoint from $A$. For every $n \in \mathbf{N}$ there exists $x_{n}$ in $Q_{n} \cap F_{n}$. The set $\left\{x_{1}, x_{2}, \ldots\right\} \cup\{x\}$ is closed, disjoint from $A$ and meeting $\mathscr{F}$. Therefore $\mathscr{F}$ does not semiconverge to $A$.

Let $A$ be a subset of a pseudotopological space $X$ and $\mathscr{F}$ a filter in $X$. The $\mathscr{F}$-boundary of $A$ is the adherence of the filter $\mathscr{F} \vee A^{c}$ if it exists and the empty set otherwise. It is denoted by $\operatorname{Fr}_{\mathscr{F}} A$.

A subset $K$ of a set $A$ is called the $\mathscr{F}$ kernel of $A$ if $\mathscr{F} \vee A^{c}$ semiconverges to $K$.

\section{THEOREM 7.5.}

(i) In a regular topological space $\operatorname{Fr}_{\mathscr{F}} A$ is a subset of every closed $\mathscr{F}$-kernel of $A$.

(ii) In a first countable (topological) space $\operatorname{Fr}_{\mathscr{F}} A$ is a subset of every $\mathscr{F}$-kernel of A provided that $\mathscr{F}$ is countably based.

Proof. If $\mathscr{F}$ is disjoint from $A^{c}$, then $\mathrm{Fr}_{\mathscr{F}} A$ is empty and the proof completed. If $\mathscr{F}$ meets $A^{c}$ then $\mathscr{F} \vee A^{c}$ semiconverges to every $\mathscr{F}$-kernel $K$ of $A$. If the space is regular, then by Theorem $4.10(\mathrm{v})$ we get (i). Part (ii) follows from Theorem 7.4 as

$$
\operatorname{Fr}_{\mathscr{F}} A=\operatorname{Adh}\left(\mathscr{F} \vee A^{c}\right) \subset K .
$$

COROLLARY 7.6. Let $\mathscr{F}$ be a countably based filter in $X$, semiconvergent to a set $A$. Assume that $X$ is metrizable. Then the F-boundary of $A$ is the minimal (and compact) $\mathscr{F}$-kernel of $A$. If $X$ is Dieudonne complete and $A$ is closed, then $\operatorname{Fr}_{\mathscr{F}} A$ is the minimal closed $\mathscr{F}$-kernel of $A$.

Proof. By Theorem 7.3, $\mathscr{F} \vee A^{c}$ is compactoid and by Proposition 4.8, $\mathscr{F} \vee A^{c}$ semiconverges to $\operatorname{Fr}_{\mathscr{F}} A$. By Theorem 7.5 the $\mathscr{F}$ boundary of $A$ is a subset of $A$, hence an $\mathscr{F}$-kernel. By the same theorem it is the minimal $\mathscr{F}$-kernel of $A$. Under any of the assumptions, $X$ is regular thus by Corollary 4.3 the kernel is compact.

Corollary 7.7. Let $\mathscr{F}$ be a countably based filter in $X$ and $A \subset X$. Assume that either $X$ is metrizable or $X$ is Dieudonné complete and $A$ is closed.

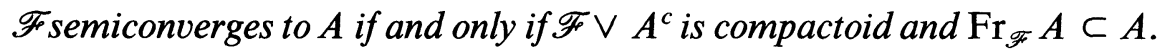

Corollary 7.6 applied to relations yields [10, Thm. 4] being a generalization of Choquet's [5, Thm. 3]. 
Corollary 7.8. Let $\Gamma: X \rightrightarrows Y$. Let $Y$ be metrizable ( $Y$ Dieudonné complete and $\Gamma x$ closed). Let $X$ be first countable at $x$. Then the active boundary of $\Gamma$ at $x$ (that is, $\Gamma \mathcal{N}(x)$-boundary of $\Gamma x)$ is the least $\Gamma \mathscr{N}(x)$ kernel, (the least closed $\Gamma \mathscr{N}(x)$-kernel) of $\Gamma x$.

As a consequence of Proposition 7.1, Corollary 4.13 and Proposition 3.18 , we quote

Proposition 7.9. ([19, Thm. 2.2]) Let $\left\{A_{t}\right\}_{t \in T}$ be a downward directed totally bounded family of nonempty closed subsets of a complete uniform space. Then $A=\bigcap_{t \in T} A_{t}$ is nonempty and compact and $\left\{A_{t}\right\}_{t \in T}$ converges to $A$ in the uniform space of nonempty closed subsets.

The Kuratowski measure of noncompactness $\alpha(A)$ of a subset $A$ of a metric space is the infimum of those $r \in \mathbf{R}_{+}$for which there is a finite partition of $A$ into subsets of diameter less than $r$ [15]. It is clear that a filter $\mathscr{F}$ in a metric space is totally bounded if and only if $\inf _{F \in \mathscr{F}} \alpha(F)=0$. As a corollary of Proposition 7.9 one gets the classical

THEOREM 7.10 [15]. Let $\left\{A_{n}\right\}_{n \in \mathbf{N}}$ be a sequence of nonempty decreasing closed subsets of a complete metric space. If $\lim _{n \rightarrow \infty} \alpha\left(A_{n}\right)=0$, then $\bigcap_{n=1}^{\infty} A_{n}$ is nonempty and compact.

8. Localization of compactness properties. With every subset $A$ of a set $X$ we associate a property $\mathfrak{P}(A)$ of subsets of $X$; in other words $\mathfrak{B}(A)$ is a family of subsets of $X(B$ has the property $\mathfrak{P}(A)$ if and only if $B \in \mathfrak{B}(A)$ ).

We assume that for every $A \subset X, \mathfrak{B}(A) \subset 2^{A}$ and $A \subset B$ implies $\mathfrak{P}(A) \subset \mathfrak{P}(B)$.

We say that a filter $\mathscr{F}$ in $X$ is locally $\mathfrak{B}$ if $\mathscr{F} \cap \mathfrak{P}(X) \neq \varnothing$. We say that $\mathscr{F}$ is hereditary locally $\mathfrak{P}$ if for every $F \in \mathscr{F}, \mathscr{F} \cap \mathfrak{B}(F) \neq \varnothing$. The latter also means that for every $F \in \mathscr{F}$ there is $F^{\prime} \subset F$ such that $F^{\prime} \in \mathscr{F} \cap \mathfrak{P}(F)$. If $\mathscr{F}$ is hereditary locally $\mathfrak{B}$, then it is locally $\mathfrak{P}$.

Call a topological space locally $\mathfrak{B}$ (hereditary locally $\mathfrak{B}$ ), if all its neighborhood filters are locally $\mathfrak{B}$ (hereditary locally $\mathfrak{B}$ ).

THEOREM 8.1. A space is hereditary locally $\mathfrak{B}$ if and only if its every open subset is locally $\mathfrak{B}$ in the induced topology.

If $\mathfrak{B}$ is the property relation "is compact in" $(B \in \mathfrak{P}(A)$ means $B$ is a compact subset of $A$ ), then $\mathscr{F}$ is locally compact (locally $\mathfrak{B}$ ) whenever it contains a compact element and $\mathscr{F}$ is hereditary locally compact (hereditary locally $\mathfrak{P})$, whenever it admits a base composed of compact sets. 
The following example (much simpler than [13, Ex. 2.2]) shows that a locally compact space need not be hereditary locally compact.

EXAMPLE 8.2. Let $\mathscr{2}$ be the space of rationals. The topology is given by

$$
\mathscr{N}(x)= \begin{cases}\{\mathscr{Q}\} & \text { if } x=0 \\ \text { usual, } & \text { otherwise }\end{cases}
$$

The space is compact (every filter converges to 0 ) thus locally compact. It is not hereditary locally compact because $\mathscr{Q} \backslash\{0\}$ is an open subset for which the induced topology is not locally compact.

If $\mathfrak{B}$ is the property relation "is compactoid in", then $\mathscr{F}$ is locally $\mathfrak{B}$ (locally compactoid) if and only if there is a compactoid set in $\mathscr{F}$ (equivalently, there is a base of $\mathscr{F}$ composed of compactoid sets). Locally compactoid spaces [5] are precisely "locally bounded spaces" of [16].

For the same property relation, $\mathscr{F}$ is hereditary locally $\mathfrak{P}$ (hereditary locally compactoid) if for every $F \in \mathscr{F}$ there is $F^{\prime} \in \mathscr{F}$ which is compactoid in $F$. Hereditary locally compactoid spaces called "quasi locally compact spaces" by Ward are equivalently defined (see [16]) by:

for every point $x$ and every neighborhood $A$ of $x$ there is a neighborhood $B \subset A$ of $x$ such that every open cover of $A$ has a finite subfamily that covers $B$.

Example 8.2 shows that a locally compactoid space need not be hereditary locally compactoid.

A locally compact space is locally compactoid; an hereditary locally compact space is hereditary locally compactoid.

Locally compactoid filters are compactoid. The following generalizes [20, Prop. 12].

Proposition 8.3. Every compactoid filter in a locally compactoid space is locally compactoid.

Proof. Follows from Theorem 3.7, since the whole space is overcoverable by compactoid sets.

Local compactness and local compactoidness coincide in subregular spaces. Hereditary local compactness and hereditary local compactoidness 
coincide in regular spaces. More precisely,

Proposition 8.4. The following properties of a topological space $X$ are equivalent:

(i) Every point of $X$ has a compact closed neighborhood.

(ii) $X$ is locally compact and subregular.

(iii) $X$ is locally compactoid and subregular.

Proof. (i) $\Rightarrow$ (ii). Every $\overline{\mathscr{N}(x)}$ contains a compact set, thus is a compactoid filter. Thus $X$ is subregular. (iii) $\Rightarrow$ (i). Every neighborhood filter $\mathscr{N}(x)$ contains a compactoid set $A_{x}$. By Corollary 4.7 the closure of $A_{x}$ is compact and closed.

Proposition 8.5. The following properties of a topological space $X$ are equivalent:

(i) Every neighborhood filter of $X$ has a base composed of closed compact sets.

(ii) $X$ is hereditary locally compact and regular.

(iii) $X$ is hereditary locally compactoid and regular.

Proof. A consequence of Theorem 8.1 and Propositions 8.4 and 4.9.

Subregularity in Proposition 8.4 may be replaced by the condition (8.1) the closure of every compactoid set is compact.

Schnare [22] shows that the following similar condition,

(8.2) the closure of every compact set is compact,

amounts to (i) of Proposition 8.4 in locally compact spaces. However, in general, in locally compactoid space, (8.2) does not imply (i) (of Proposition 8.4), since in [16, Ex. 1] there is a Hausdorff locally compactoid space which is not locally compact.

It is important to note that in regular spaces all properties of Propositions 8.4 and 8.5 are equivalent. Indeed, one has

Proposition 8.6. If a regular space is locally compactoid, then it is hereditary locally compactoid.

To this we add information on regular spaces.

Proposition 8.7. If a Hausdorff space is either locally compact or hereditary locally compactoid, then it is regular. 
[16, Ex. 2] shows that in Proposition 8,7 hereditary local compactoidness must not be replaced by local compactoidness.

9. Closure of composed relations. As an example of applications consider topological spaces $X, Y, Z$ and relations $\Gamma: X \rightrightarrows Y$ and $\Delta$ : $Y \rightrightarrows Z$. Their composition is the relation $\Delta \Gamma: X \rightrightarrows Z$ defined by

$$
\Delta \Gamma x=\bigcup_{y \in \Gamma x} \Delta y .
$$

The composition of graph-closed relations need not be graph-closed. However, we have

Proposition 9.1. Let $\Gamma, \Delta$ be graph-closed. If for every $(x, z)$ in the closure of $\Delta \Gamma$ the filter $\Delta^{-1} \mathcal{N}(z) \vee \Gamma \mathcal{N}(x)$ has nonempty adherence, then $\Delta \Gamma$ is graph-closed.

Proof. If $(x, z) \in \mathrm{Cl}(\Delta \Gamma)$, then for every $Q \in \mathcal{N}(x)$ and $W \in \mathcal{N}(z)$ we have $Q \times W \cap \Delta \Gamma \neq \varnothing$; in other words, $\Delta^{-1} W \cap \Gamma Q \neq \varnothing$. Consequently, the filter $\Delta^{-1} \mathscr{N}(z) \vee \Gamma \mathscr{N}(x)$ exists and, by assumption,

$$
\begin{aligned}
\varnothing & \neq \operatorname{Adh}\left(\Delta^{-1} \mathscr{N}(z) \vee \Gamma \mathscr{N}(x)\right) \subset \operatorname{Adh} \Delta^{-1} \mathscr{N}(z) \cap \operatorname{Adh} \Gamma \mathscr{N}(x) \\
& \subset \Delta^{-1} z \cap \Gamma x .
\end{aligned}
$$

It follows that $(x, z) \in \Delta \Gamma$.

Corollary 9.2. Let $\Gamma$ and $\Delta$ be graph-closed. If either $\Gamma$ or $\Delta^{-1}$ is subcontinuous, then $\Delta \Gamma$ is graph-closed.

Consider the special case in which $\Delta$ is the epigraph of an extended real-valued function $f: Y \rightarrow \overline{\mathbf{R}}(\Delta=$ epi $f$ ). The composition (epi $f$ ) $\circ \Gamma$ is graph-closed if and only if the marginal function $f \Gamma(f \Gamma(x)=$ $\left.\inf _{y \in \Gamma x} f(y)\right)$ is lower semicontinuous and $f$ attains its infimum on every $\Gamma x$ (see [6]). The epigraph of $f$ is graph-closed if and only if $f$ is lower semicontinuous.

COROLlaRy 9.3. Let $f: Y \rightarrow \overline{\mathbf{R}}$ be a lower semicontinuous function and $\Gamma: X \rightrightarrows Y$ a graph-closed subcontinuous relation. Then the marginal function $f \Gamma$ is lower semicontinuous and $f$ attains its infimum on every $\Gamma x$.

Note that we know that $\Gamma$ is upper semicontinuous and closed-valued, but for nonsubregular spaces we may not have compact values of $\Gamma$.

A function $f$ is well-conditioned if it is lower semicontinuous and its level relation (epi $f)^{-1}$ is subcontinuous. This definition is equivalent to [20, Def. 16] and is less stringent than that of well-posedness. 
COROLlaRY 9.4. If $f$ is well-conditioned and $\Gamma$ is graph-closed, then $f \Gamma$ is lower semicontinuous and $f$ attains its infimum on every $\Gamma x$.

The above is not surprising, since a well-conditioned function attains its infimum on every closed set. For other conditions on lower semicontinuity of marginal function see [10].

Another special case is that of linear (graph-) closed operations $\Gamma, \Delta$ in topological vector spaces. Then the subcontinuity of $\Delta^{-1}$ implies that $\Delta^{-1}$ is an operator.

COROllary 9.5. Let $\Gamma, \Delta$ be graph-closed linear operators. $\Delta \Gamma$ is graph-closed if either $\Gamma$ is continuous or $\Delta$ has the continuous inverse.

\section{REFERENCES}

[1] R. Arens and J. Dugundji, Topologies for function spaces, Pacific J. Math., 1 (1951), 5-31.

[2] J. Banaś and K. Goebel, Measures of Noncompactness in Banach Spaces, Lecture Notes in Pure and Appl. Math., M. Dekker, New York-Basel, 1980.

[3] E. Čech, Topological Spaces, Czechoslovak Academy of Sciences, Prague, Interscience Publishers, London-New York-Sydney, 1966.

[4] G. Choquet, Sur les notions de filtre et de grille, C. R. Acad. Sci. Paris, 224 (1947), 171-173.

[5] _ Convergences, Annales Université de Grenoble, 23 (1947-1948), 55-112.

[6] S. Dolecki, Abstract study of optimality conditions, J. Math. Anal. Appl., 73 (1980), 24-48. Corrigendum, 82 (1981), 295-296.

[7] S. Dolecki and G. H. Greco, Cyrtologies of convergences, I, Math. Nachr., to appear.

[8] _ Familles pseudotopologiques de filtres et compacité, C. R. Acad. Sci. Paris, 296 (1983), 211-214.

[9] S. Dolecki and A. Lechicki, Semi-continuité supérieure forte et filtres adhérents, C. R. Acad. Sci. Paris, 293 (1981), 219-221.

[10] On structure of upper semicontinuity, J. Math. Anal. Appl., 88 (1982), 547-554.

[11] R. Engelking, General Topology, Monografie Matematyczne 60, PWN-Polish Scientific Publishers, Warsaw, 1977.

[12] W. Gähler, Grundstrukturen der Analysis, I, Birkhäuser Verlag, Basel, Stuttgart,1977.

[13] J. L. Gross, A third definition of local compactness, Amer. Math. Monthly, 74 (1967), 1120-1122.

[14] R. Hrycay, Noncontinuous multifunctions, Pacific J. Math., 35 (1970), 141-154.

[15] K. Kuratowski, Topology, Vol. I, II, Academic Press, New York, 1966-1968.

[16] P. Th. Lambrinos, Locally bounded spaces, Proc. Edinburgh Math. Soc., 19 (19741975), 321-325.

[17] A. Lechicki, Some problems in the theory of continuous and measurable multifunctions (in Polish), Thesis, University of Poznan, 1980.

[18] _ On bounded and subcontinuous multifunctions, Pacific J. Math., 75 (1978), 191-197.

[19] , A generalized measure of noncompactness and Dini's theorem for multifunctions, to appear. 
[20] J. P. Penot, Compact nets, filters and relations, J. Math. Anal. Appl., 93 (1983), 400-417.

[21] C. T. Scarborough, Minimal Urysohn spaces, Pacific J. Math., 27 (1968), 611-617.

[22] P. S. Schnare, Two definitions of local compactness, Amer. Math. Monthly, 72 (1965), 764-765.

[23] R. E. Smithson, Subcontinuity for multifunctions, Pacific J. Math., 61 (1975), 283-288.

[24] F. Topsøe, Compactness in spaces of measures, Studia Math., 36 (1970).

[25] P. Urysohn, Sur les classes (L) de M. Fréchet, Ens. Math., 25 (1926), 77-83.

Received February 14, 1983 and in revised form August 8, 1983. The first author's work was partially supported by the Consiglio Nationale delle Ricerche.

POLISH ACADEMY OF SCIENCES

WARSAW, POLAND AND UNIVERSITA

di TRENTo, Povo, Italy

AND

UNIVERSITA DI TRENTO

38050 Povo (TRENTo) Italy

AND

Pedagogical University

SzCZECIN, POLAND 


\section{PACIFIC JOURNAL OF MATHEMATICS EDITORS}

DONALD BABBITT (Managing Editor)
University of California
Los Angeles, CA 90024
CHARLES R. DePrima
California Institute of Technology
Pasadena, CA 91125
R. FinN
Stanford University
Stanford, CA 94305

HERMANN FLASCHKA

University of Arizona

Tucson, AZ 85721

RAMESH A. GANGOLLI

University of Washington

Seattle, WA 98195

ROBION KIRBY

University of California

Berkeley, CA 94720

C. C. MOore

University of California

Berkeley, CA 94720
Hugo RossI

University of Utah

Salt Lake City, UT 84112

H. SAMELSON

Stanford University

Stanford, CA 94305

HaRold STARK

University of California, San Diego

La Jolla, CA 92093

\section{ASSOCIATE EDITORS}
R. ARENS
E. F. BECKENBACH (1906-1982)
B. H. NEUMANN
F. WOLF
K. YoshidA

\section{SUPPORTING INSTITUTIONS}

UNIVERSITY OF ARIZONA

UNIVERSITY OF BRITISH COLUMBIA

CALIFORNIA INSTITUTE OF TECHNOLOGY

UNIVERSITY OF CALIFORNIA

MONTANA STATE UNIVERSITY

UNIVERSITY OF NEVADA, RENO

NEW MEXICO STATE UNIVERSITY

OREGON STATE UNIVERSITY
UNIVERSITY OF OREGON

UNIVERSITY OF SOUTHERN CALIFORNIA

STANFORD UNIVERSITY

UNIVERSITY OF HAWAII

UNIVERSITY OF TOKYO

UNIVERSITY OF UTAH

WASHINGTON STATE UNIVERSITY

UNIVERSITY OF WASHINGTON 


\section{Pacific Journal of Mathematics}

\section{Vol. 117, No. $1 \quad$ January, 1985}

Amos Altshuler and Leon Steinberg, The complete enumeration of the

4-polytopes and 3 -spheres with eight vertices $\ldots \ldots \ldots \ldots \ldots \ldots \ldots \ldots$

Michael James Beeson, The $6 \pi$ theorem about minimal surfaces . . . . . . . . 17

Jeffrey Lawrence Caruso and Stefan Waner, An approximation theorem

for equivariant loop spaces in the compact Lie case ...............27

Jo-Ann Deborah Cohen, Topologies on the quotient field of a Dedekind

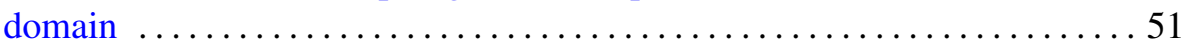

Szymon Dolecki, Gabriele H. Greco and Alois Andreas Lechicki,

Compactoid and compact filters .........................6 69

Roger William Hansell (Sr.), Generalized quotient maps that are

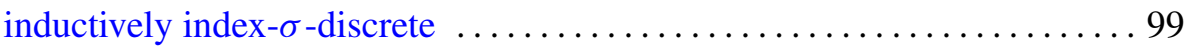

Gerhard Huisken, Capillary surfaces over obstacles $\ldots \ldots \ldots \ldots \ldots \ldots \ldots 121$

Jun Shung Hwang, A problem on continuous and periodic functions . . . . 143

Ronald Fred Levy and Michael David Rice, The extension of equi-uniformly continuous families of mappings $\ldots \ldots \ldots \ldots \ldots \ldots 149$

Kevin Mor McCrimmon, Derivations and Cayley derivations of generalized

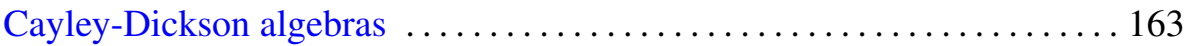

H. M. (Hari Mohan) Srivastava, A multilinear generating function for the Konhauser sets of biorthogonal polynomials suggested by the Laguerre

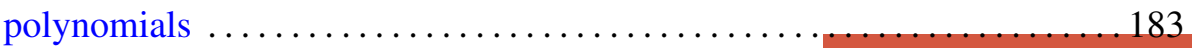

Zhu Jia Lu, Some maximum properties for a family of singular hyperbolic operators 